\title{
Measurement and Analysis of Current Signals for Gearbox Fault Recognition of Wind Turbine
}

\author{
Deng-Fa Lin ${ }^{1}$, Po-Hung Chen ${ }^{1}$, Mike Williams ${ }^{2}$ \\ ${ }^{1}$ Department of Electrical Engineering, St. John’s University, Taipei 25135, Taiwan \\ dflin@mail.sju.edu.tw, phchen@mail.sju.edu.tw \\ ${ }^{2}$ Managing Director of Central Data Control Ltd., London SE1 7PB, United Kingdom \\ mike.williams@cdc.uk.com
}

\begin{abstract}
This paper presents a novel solution method based on measurement and analysis of current signals for gearbox fault recognition of wind turbine. A gearbox with typical oil-leakage fault is purposely made. The oil-leakage gearbox and a normal gearbox are used as experimental models to measure and analyze the current signals of generator. This work employs wavelet transform (WT), empirical mode decomposition (EMD) and fast Fourier transform (FFT) to analyze the current signals for both the oil-leakage and the normal gearboxes. K-nearest neighbors $(\mathrm{KNN})$ is used on automatic fault recognition. First, the normal gearbox and the oilleakage gearbox are separately applied to practical power platform experiments. Second, empirical mode decomposition is applied on analyzing the intrinsic mode function (IMF) of the current signals, and fast Fourier transform is used to get the intrinsic mode function spectrum. Finally, the features of the spectrum are extracted, and K-nearest neighbors is used on gearbox fault recognition of wind turbine. Experimental results indicate that the proposed solution method can effectively recognize the oilleakage fault of gearboxes.
\end{abstract}

Keywords: Wavelet transform, empirical mode decomposition, fast Fourier transform, K-nearest neighbors, intrinsic mode function.

\section{INTRODUCTION}

$\mathrm{A}$ S THE RESOURCES in the world are finite, green energy has become an inevitable trend. Installations of green energy equipments are on the rise, but so too are the fault occurrences of said installations. Wind turbines are widely used now; not only are they very costly but they also require many components. In particular, the gearbox determines whether a turbine malfunctions or not. Gearboxes with broken gear-tooth or inadequate lubrication might lead to vibration or high temperatures, which might damage the wind turbines [1]-[2].

To stabilize the performance of wind turbines, the solution is to monitor the status of each component. According to Bussel and Schontag [3], an accelerometer can measure the vibration signals of a running gearbox to investigate its status. However, this approach is costly and takes considerable manpower. Furthermore, the results tend to be affected by external interference. Therefore, this paper analyzes the currents signals of wind generators instead of the vibration signal, which can reduce the recognition time and maintenance cost.

First, this work measures current signals of the wind turbine with an oil-leakage gearbox. Second, the current signals are decomposed into four layers (from high frequency to low frequency) by wavelet transform (WT) [4][7] and empirical mode decomposition (EMD) [8]-[11]. Then, those signals are transformed by fast Fourier transform (FFT) [12]-[13] and illustrate a spectrum. Third, the features of the spectrum are extracted. Finally, K-nearest neighbors [14]-[16] is employed on fault classification. This method can have the same effect as vibration signal analysis without incurring additional labor costs and expensive sensors.
As FFT can not analyze transient signals, analysis will occur after EMD to achieve the purpose of analyzing current signals. In addition, EMD can decompose input signals according to different frequencies and can effectively avoid interferences from noise, which will help the signal's analysis. Finally, the paper uses KNN to classify current signals. Because of KNN's simple structure and fast operation, it precludes the need for prior training on the part of maintenance personnel.

The purpose of this study is to propose a method recognizing the fault signals of an oil-leakage wind turbine. Regular maintenance only avoids the occurrence of a breakdown, and early detection of faults can be a costsaving and preventative measure.

\section{SUBJECT \& METHODS}

In this paper, a test power platform with an $11 \mathrm{kw}$ motor (Fig.1.) is used to drive the gearbox with a $360 \mathrm{rpm}$ rated speed generator. This test power platform simulates a small wind turbine. The measurement of the current signals of the generator is described as follows:

Step 1: To connect the load to the output of the generator.

Step 2: To series one phase to signal capture device, which is called PXI-1033. Then use the sensor to observe the range of the current. The signal capture device is connected to personal computer for signal segmentation and storage.

Step 3: To open the motor and let it drive the gearbox and the generator and then measure the signal (Fig.2.).

To simulate an oil leakage fault of gearbox, the following steps are then taken. First, the oil that is in the gearbox of the wind turbine is exposed. Second, the gearbox on the wind turbine is replaced, and then current signals of the oil leakage fault are obtained. In this paper, the anomalistic 
types are defined as the oil leakage of the gearbox. Third, sample the normal types and anomalistic types at one thousand times per unit.

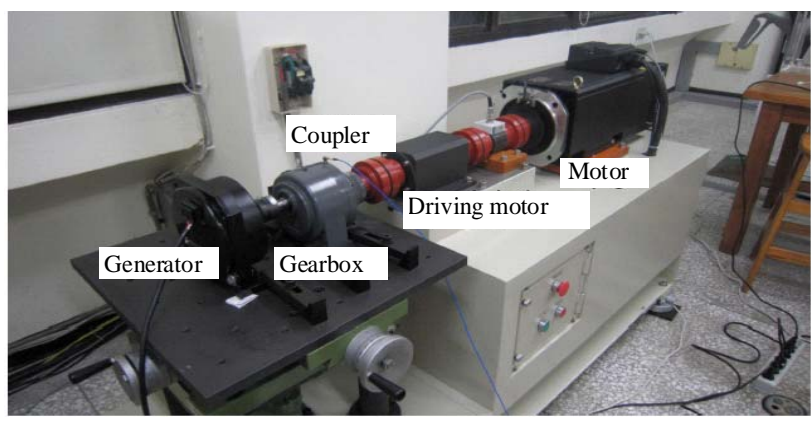

Fig.1. Test power platform

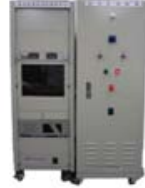

control the motor

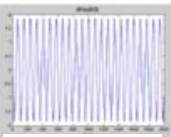

produce the intput signal

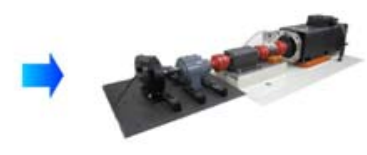

use the motor to drive the genertor

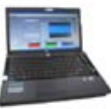

save and divide the signal produce the current signal

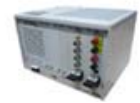

capture the current signal
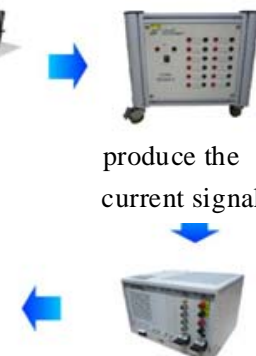

Fig.2. Measurement of current signals

\section{TEST RESULTS AND DISCUSSION}

First, this paper adopts a dynamometer test bed to obtain the current signals of both normal and oil-leakage gearboxes. Second, EMD is used to decompose the signals based on different frequencies. Third, the high-frequency signals on the first layer are transformed by FFT and illustrate a spectrum. Then, the features of the spectrum are extracted for training. Finally, KNN is employed to recognize the status of the gearbox. The solution process is shown in Fig.3.

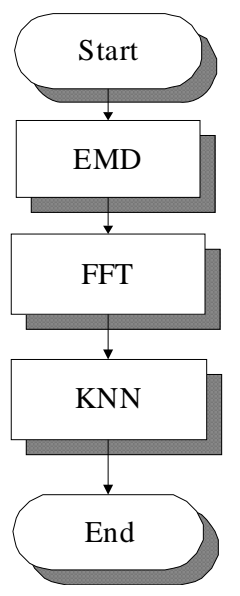

Fig.3. Solution process
The output current signals of generator with both normal and damaged gearboxes are measured. The Hilbert spectrums of the output currents of both normal and damaged gearboxes are illustrated in Fig.4. and Fig.5. In the Fig.4., the spectrum is clear with a stable frequency component and a measurement noise. However, the disturbance phenomena exit within 10 to $50 \mathrm{~Hz}$ and 100 to $130 \mathrm{~Hz}$ in the spectrum of Fig.5.

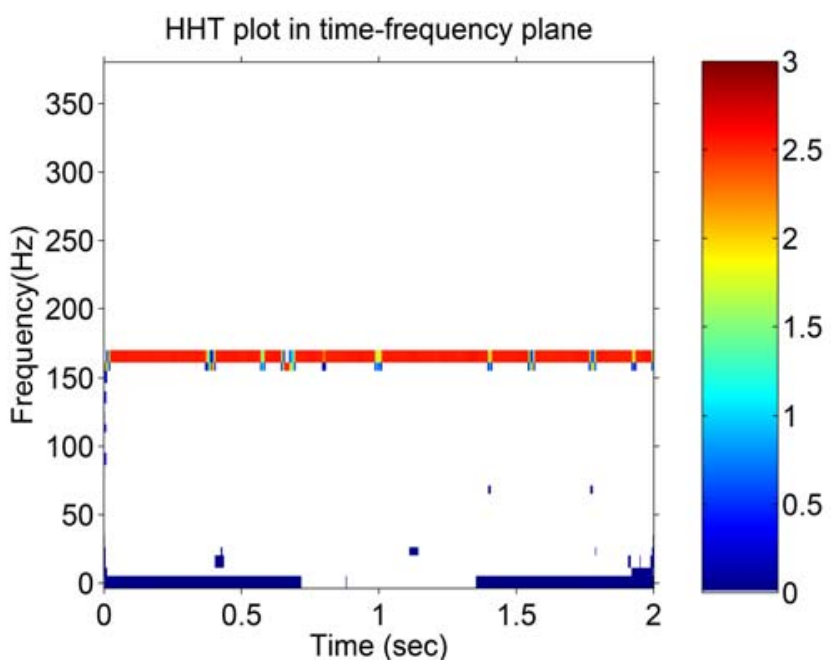

Fig.4. Measured current signals of normal gearbox

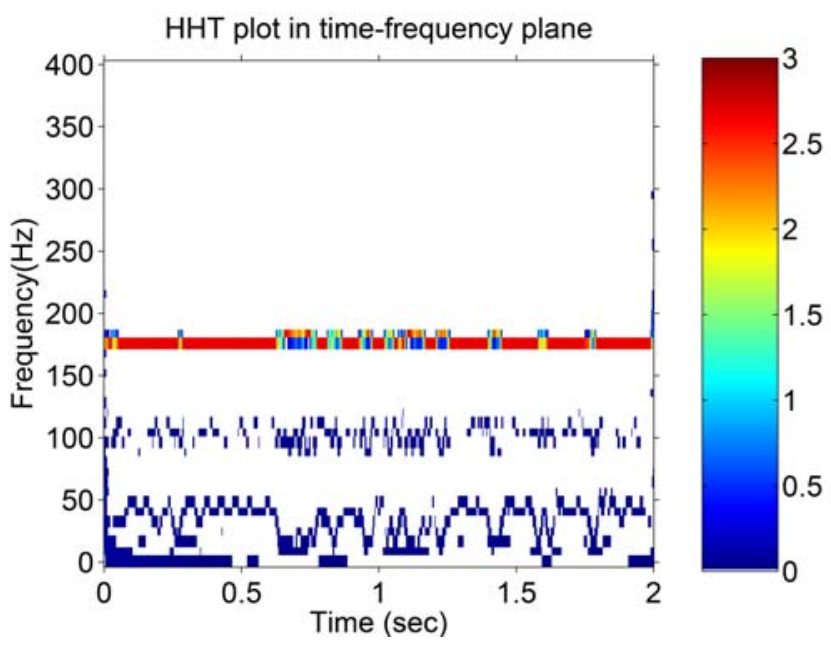

Fig.5. Measured current signals of oil-leakage gearbox

\section{A. Empirical Mode Decomposition (EMD)}

Fig.6. shows the general flow chart of the EMD. The input current signal of the generator $\mathrm{x}(\mathrm{t})$ is captured as shown in Fig.7. and this signal has a maximum and a minimum. Then apply EMD to this signal, which are described as following steps.

Step 1: To find the maximum area and the minimum area of the input signal, as shown in Fig.8.

Step 2: Use Cubic spline interpolation to connect the extreme points to obtain the regional maximum envelope line and minimum envelope line, as shown in Fig.9. This method is a form of interpolation. It avoids the problem of Runge's phenomenon. Cubic curve is defined as in (1)-(3). 


$$
\begin{gathered}
S_{i}(x)=\frac{z_{i+1}\left(x-x_{i}\right)^{3}+z_{i}\left(x_{i+1}-x\right)^{3}}{6 h_{i}} \\
+\left(\frac{y_{i+1}}{h_{i}}-\frac{h_{i}}{6} z_{i+1}\right)\left(x-x_{i}\right)+\left(\frac{y_{i}}{h_{i}}-\frac{h_{i}}{6} z_{i}\right)\left(x_{i+1}-x\right) \\
h_{i}=x_{i+1}-x_{i}
\end{gathered}
$$$$
\left\{\begin{array}{l}
z_{0}=0 \\
h_{i-1} \times z_{i-1}+2 \times\left(h_{i-1}+h_{i}\right) \times z_{i}+h_{i} \times z_{i+1} \\
=6 \times\left(\frac{y_{i+1}-y_{i}}{h_{i}}-\frac{y_{i}-y_{i-1}}{h_{i-1}}\right) \\
z_{n}=0
\end{array}\right.
$$$$
\text { for } i=1,2, \ldots n-1
$$

Step 3: Envelope of the two extreme points in the sum is divided by two, such as in (4), can obtain the mean curve, as shown in Fig.9.

$$
m_{1}(t)=\frac{\max \operatorname{line}(t)+\min \text { line }(t)}{2}
$$

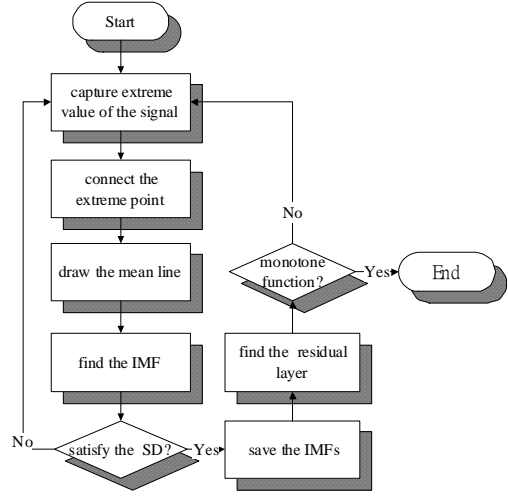

Fig.6. General flow chart of EMD

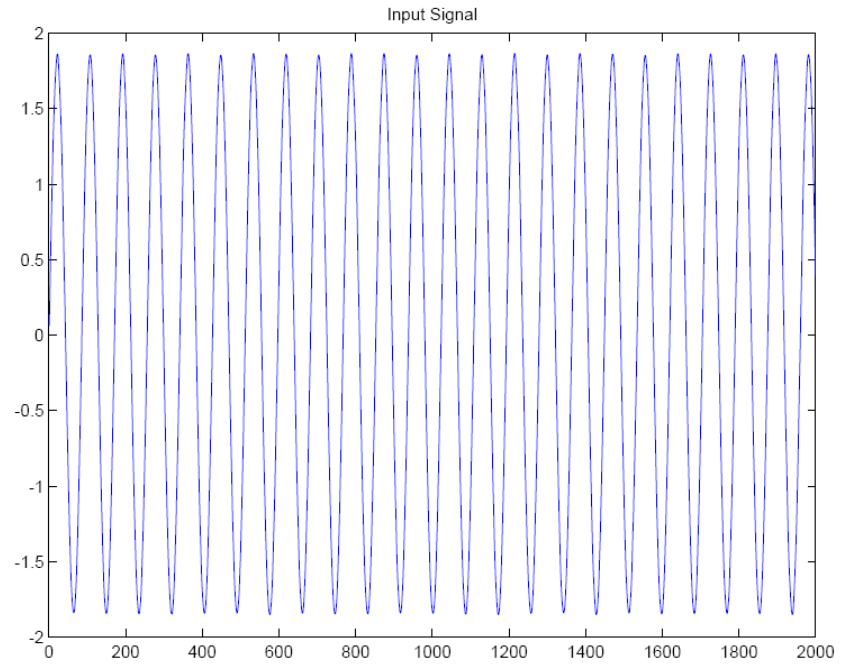

Fig.7. Input signal of EMD

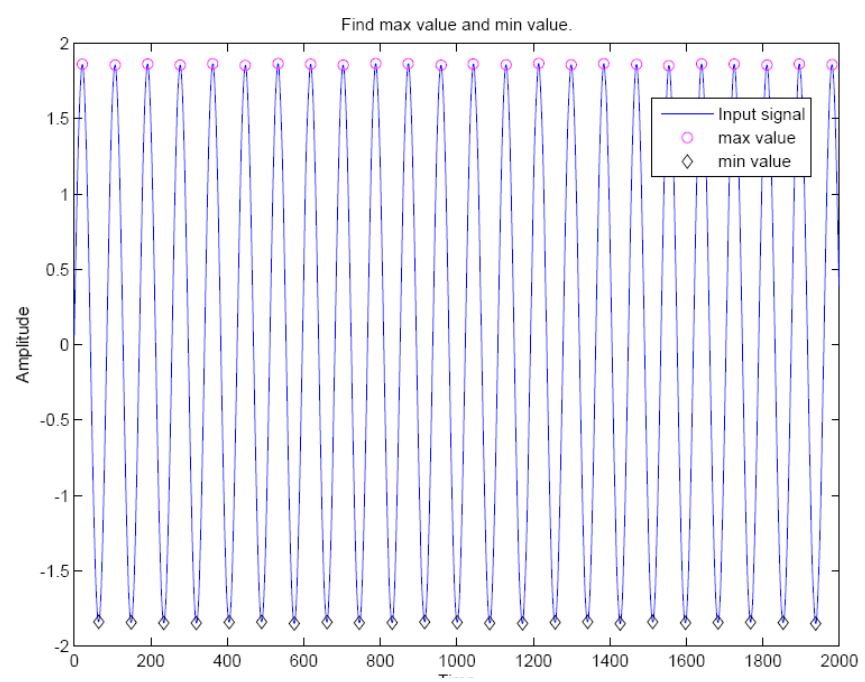

Fig.8. Maximum and minimum areas of signal

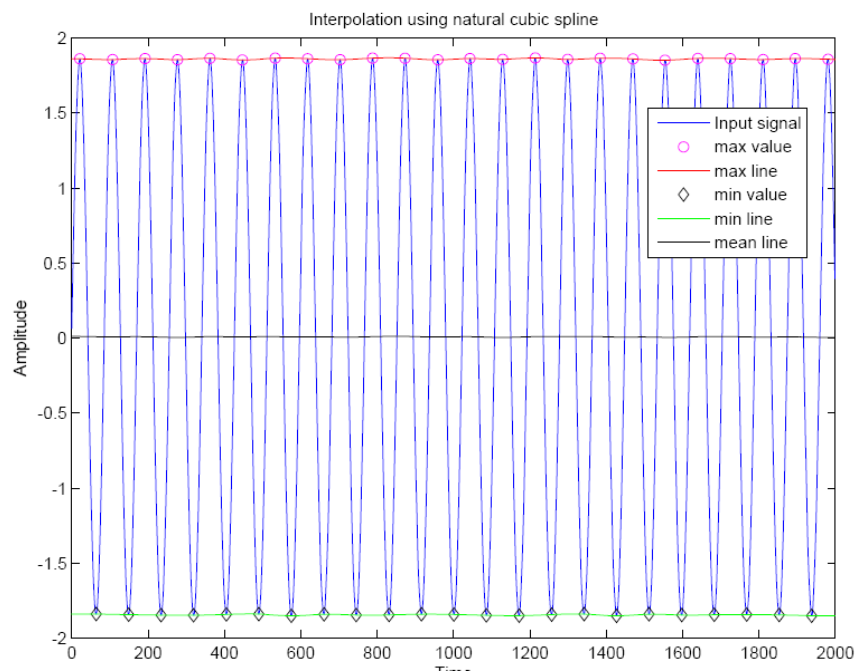

Fig.9. Envelope line and mean line

Step 4: Connect them to produce the upper and lower $x(t)$ minus the mean line $m(t)$ is $h_{1}(t)$, and use $h_{1}(t)$ to repeat step $1 、 2 、 3$ can obtain $m_{11}(t)$. $h_{1}(t)$ minus the mean line $m_{11}(t)$ is $h_{11}(t)$. And so on, this recursive calculation computes to the end as in (5). The formula SD to determine this condition as in (6). Then assume $h_{1 k}(t)$ to $c_{1}(t)$, that is called IMF as in (7).

$$
\left\{\begin{array}{c}
h_{1}(t)=x(t)-m_{1}(t) \\
h_{11}(t)=h_{1}(t)-m_{11}(t) \\
\vdots \\
h_{1 k}(t)=h_{1(k-1)}(t)-m_{1 k}(t)
\end{array}\right.
$$




$$
\begin{gathered}
S D_{k}=\frac{\sum_{t=0}^{T}\left|h_{k-1}(t)-h_{k}(t)\right|^{2}}{\sum_{t=0}^{T} h_{k-1}^{2}} \\
C_{1}(t)=h_{1 k}
\end{gathered}
$$

The numbers of IMF are finite after decomposition, and the frequency is gradually decreasing. IMF must satisfy the conditions as follows:

Condition 1: The difference of the number of the zero-crossing is and the extreme point is less than one.

Condition 2: The maximum line and the minimum line are symmetrical to the mean line.

Step 5: $x(t)$ minus $c_{1}(t)$ can obtain residual value $r_{1}(t)$, as in (8). This recursive is end to $r_{n}(t)$. Then determine $r_{n}(t)$ to a monotone function as in (9). Finally, IMFs and residual layer can be obtained as showed in Fig.10.

$$
\begin{gathered}
r_{1}(t)=x(t)-c_{1}(t) \\
\left\{\begin{array}{c}
r_{2}(t)=r_{1}(t)-c_{1}(t) \\
\vdots \\
r_{n}(t)=r_{n-1}(t)-c_{n}(t)
\end{array}\right.
\end{gathered}
$$

In the theory, the input signal will be same as the sum of the IMFs and residual layer as in (10) and Fig.11.

$$
x(t)=\sum_{j=1}^{n} c_{j}(t)+r_{n}(t)
$$

\section{B. Fast Fourier Transform (FFT)}

This method is the numerical analysis of the Discrete Fourier Transform valid tool for spectrum analysis [12][13]. Compared to the Fourier transform, it has lower computational complexity.

As shown in Fig.12, use FFT to IMF and residual layer can obtain the frequency domain of the signal.

\section{K-nearest Neighbors (KNN)}

K-nearest neighbors is the simplest unsupervised learning method of the machine learning. It estimates the similarity between feature vectors of the known and unknown event in the feature vector space.

This paper uses the Euclidean Distance to measure its similarity of the vector. Consider the K-nearest known event's feature vector and by the way of majority vote to determine the unknown type of the event.
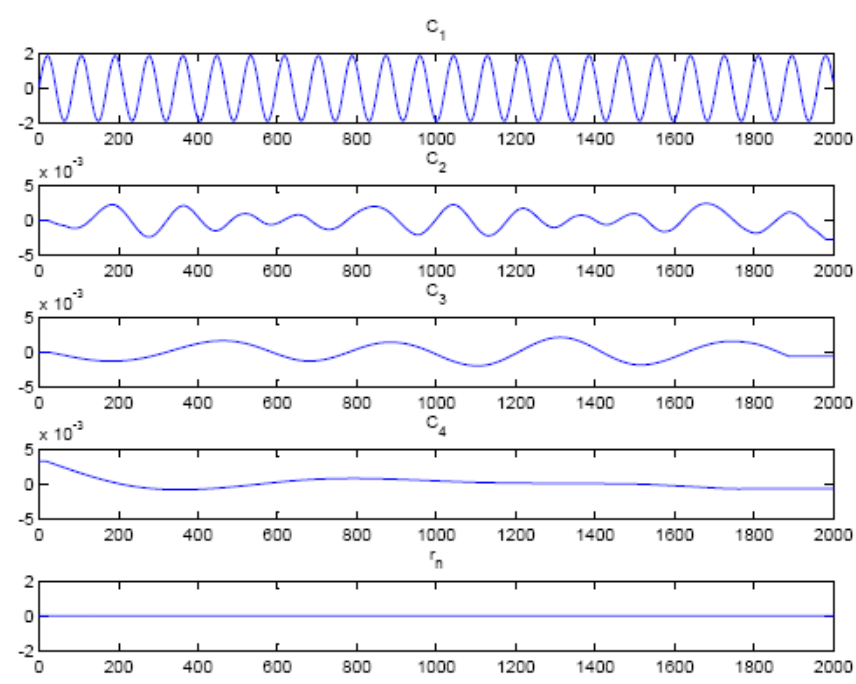

Fig.10. IMFs and residual layer

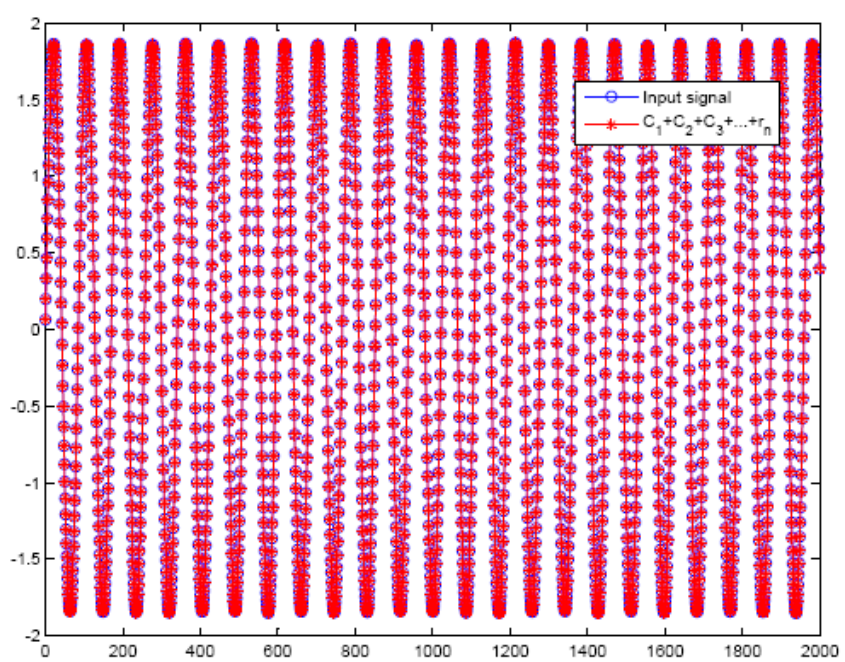

Fig.11. Sum of IMFs and residual layer
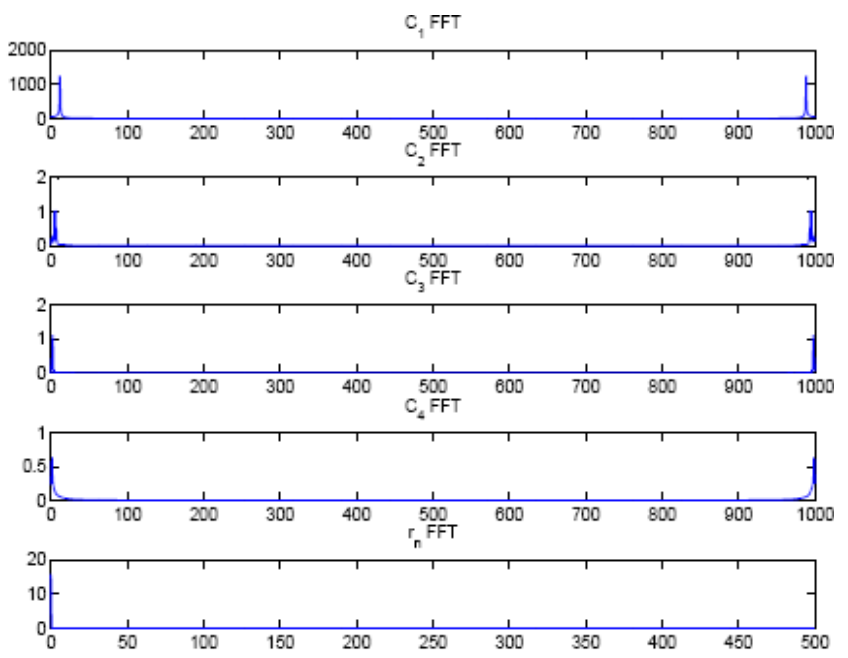

Fig.12. Results of IMF and residual layer's FFT 


\section{CONCLUSIONS}

A breakdown of gearbox could cause an unplanned outage of wind turbine and result in a loss of considerable money. This paper proposes a new approach for gearbox fault recognition of wind turbine based on measurement and analysis of current signals without any additional apparatus such as accelerometer or oil-leakage detector. An oilleakage gearbox and a normal gearbox are used as the experimental models. In this work, the analysis of current signals is a hybrid approach using WT, EMD, FFT, and KNN algorithms. In the proposed approach, the current signals of both oil-leakage and normal gearboxes are decomposed into four layers signals by the WT. The EMD is used to decompose the current signals based on different frequencies. The FFT is mainly applied to obtain the IMF's spectrums of the generator currents. The KNN is advantageous to the recognition of spectrums of current signals. The proposed approach is successfully applied to practical power platform experiments. Experimental results show that the hybrid algorithms are advantageous to the recognition of the oil-leakage fault of gearbox through measurement and analysis of generator current.

\section{ACKLOWLEDGMENT}

The support of this research by the National Science Council of the Republic of China, Taiwan under Grant NSC-99-2622-E-129-004-CC3 is gratefully acknowledged.

\section{REFERENCES}

[1] Lu, W., Jing, W., Yuan, G., Yan, L. (2013). A gearbox fault diagnosis scheme based on near-field acoustic holography and spatial distribution features of sound field. Journal of Sound and Vibration, 332 (10), 25932610.

[2] Lu, Y., Tang, J. Luo, H. (2012). Wind turbine gearbox fault detection using multiple sensors with features level data fusion. Journal of Engineering for Gas Turbines and Power, 134 (4), 907-914.

[3] Bussel, V., Schontag, C. (1997). Operation and maintenance aspects of large offshore windfarms. In European Wind Energy Conference (EWEC '97), 6-9 October 1997, 272-275.

[4] Hussain, M.S., Mamun, M. (2012). Effectiveness of the wavelet transform on the surface EMG to understand the muscle fatigue during walk. Measurement Science Review, 12 (1), 28-33.

[5] Phinyomark, A., Limsakul, C., Phukpattaranont, P. (2011). Application of wavelet analysis in EMG feature extraction for pattern classification. Measurement Science Review, 11 (2), 45-52.
[6] Stojanovic, R., Karadaglic, D., Mirkovic, M., Milosevic, D. (2011). A FPGA system for QRS complex detection based on integer wavelet transform. Measurement Science Review, 11 (4), 131-138.

[7] Janusek, D., Kania, M., Zaczek, R., Zavala-Fernandez, H., Zbiec, R., Opolski, G., Maniewski, R. (2011). Application of wavelet based denoising for T-wave alternant analysis in high resolution ECG maps. Measurement Science Review, 11 (6), 181-184.

[8] Zhang, J.C., Zhao, L. (2011). A speech denoising method based on improved EMD. IEEE Transactions on Magnetics, 2, 305-309.

[9] Peng, N., Hong, X., Yan, L., Xin, L., Zheng, L. (2011). Aviation tool wear states identifying based on EMD and SVM. IEEE Transactions on Magnetics, 246-249.

[10] Meng, H., Zeng, L., Quan, H., Yong, Z. (2010). Research of signal frequency domain resolution basedEMD. IEEE Transactions on Magnetics, 671-675.

[11] Cui, J., Zheng, X., Li, M., Li, Z., Liu, D. (2009). Health diagnosis for air-craft based on EMD and neural network. IEEE Transactions on Magnetics, 1, 677-680.

[12] Jingzhuo, S., Dianguo, X., Baoting, L. (2003). Sensorless speed detection based on STFT of seriesexcited motor. In Proceedings of the $20^{\text {th }}$ IEEE Instrumentation and Measurement Technology Conference (IMTC '03), 20-22 May 2003. IEEE, Vol. 2, 1322-1326.

[13] Wang, Y., Tang, Y., Jiang, Y., Chung, J.G., Song, S. S., Lim, M.S. (2007). Novel memory reference reduction methods for FFT implementations on DSP processors. IEEE Transactions on Magnetics, 5, 23382349.

[14] Wang, L., Khan, L., Thuraisingham, B. (2008). An effective evidence theory based K-nearest neighbor classification. IEEE Transactions on Magnetics, 1, 797-801.

[15] Jiang, Z., Deng, Y. (2010). On the time series knearest neighbor classification of abnormal brain activity. IEEE Transactions on Magnetics, 6, 10051016.

[16] Jiang, Z., Deng, Y. (2010). Improving KNN based text classifications. IEEE Transactions on Magnetics, 4, 2338-2349.

Received July 16, 2012. Accepted April 2, 2013. 